\title{
Clinical Images \\ Clinical Image: Clubbed with a Reminder to Test for HIV
}

\author{
Mona Siddiqui, MD, MPH' and Michael T. Melia, MD² \\ 'Division of General Internal Medicine, Johns Hopkins University School of Medicine, Baltimore, MD, USA; ${ }^{2}$ Division of Infectious Diseases, Johns \\ Hopkins University School of Medicine, Baltimore, MD, USA.
}

J Gen Intern Med 29(9):1308

DOI: $10.1007 / \mathrm{s} 11606-014-2852-7$

(c) Society of General Internal Medicine 2014

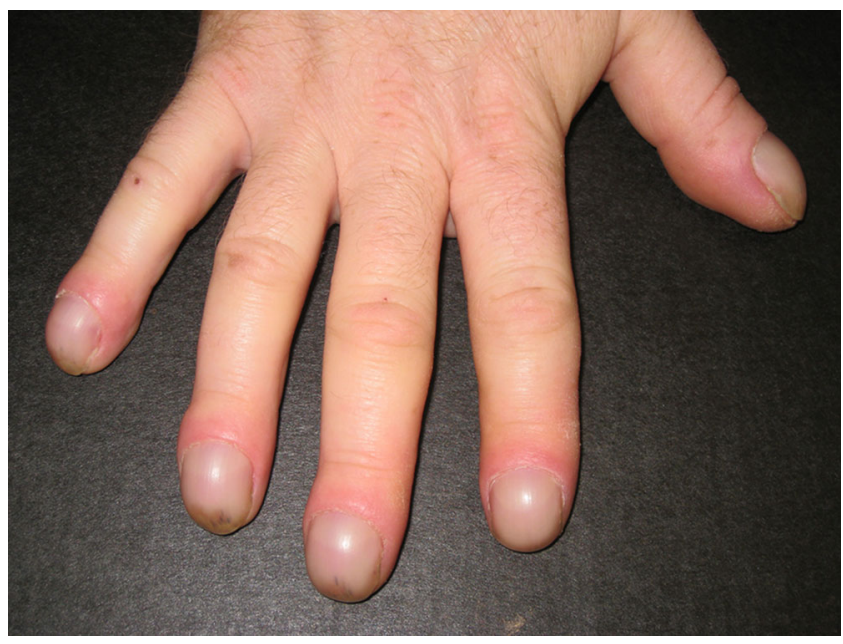

Figure 1. Digital clubbing.

\begin{abstract}
A previously healthy 34-year-old man was evaluated for two years of progressive digital clubbing. He denied fevers, night sweats, cardiopulmonary symptoms, travel, or unusual exposures. Physical examination demonstrated no pathologic lymphadenopathy, genital lesions or penile discharge. The patient had dramatic clubbing of all digits and erythematous nail beds (Figs. 1 and 2).

There are many different diseases associated with clubbing. Pulmonary diseases are most commonly associated, but cardiac conditions, gastrointestinal diseases, and various infections are also found with clubbing. The patient's evaluation included unremarkable labs for systemic diseases such as malignancy, liver disease, and inflammatory bowel disease. His cardiopulmonary evaluation included echocardiography, pulmonary function testing, polysomnography and chest computed tomography. These were also unrevealing. The patient's PPD test and RPR were nonreactive. His HIV antibody test (with Western blot)
\end{abstract}

Received September 9, 2013

Revised November 4, 2013

Accepted March 18, 2014

Published online April 15, 2014

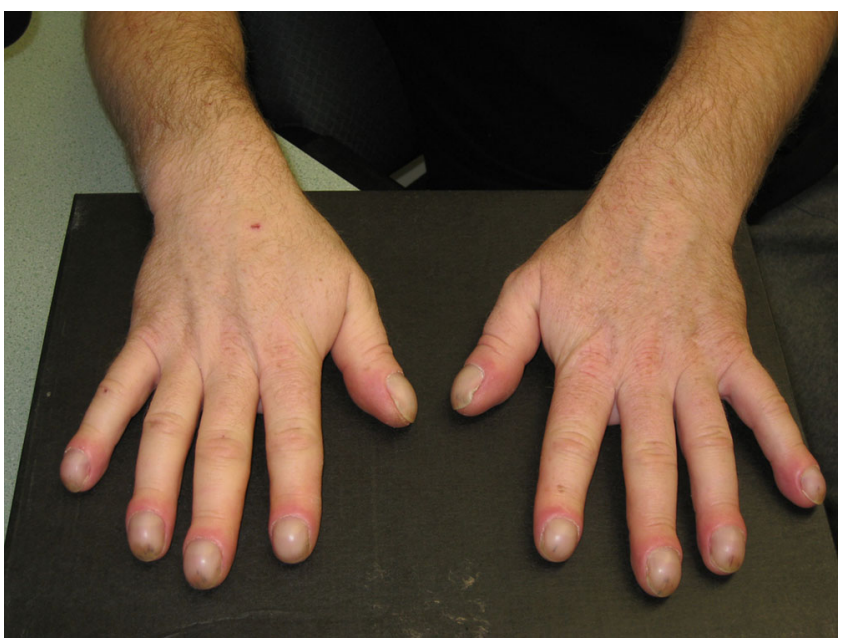

Figure 2. Digital clubbing.

returned positive. CD4+ lymphocyte count was $401 / \mu \mathrm{L}$ and viral load was 205,000 copies $/ \mathrm{mL}$.

Digital clubbing is associated with HIV. Among one convenience sample of $76 \mathrm{HIV}$-infected patients, 28 (36\%) had objectively confirmed clubbing - thus, HIV testing is recommended as part of a diagnostic algorithm. ${ }^{1,2}$ While HIV screening is now recommended for all patients aged 15-65 years, this case highlights the need for HIV diagnostic testing in patients with digital clubbing. ${ }^{3}$

Acknowledgements: Dr. Siddiqui's salary was supported by an AHRQ NRSA Comparative Effectiveness Development Training Award (1T32HSO19488-02).

Conflicts of Interest: The authors declare that they do not have a conflict of interest.

Corresponding Author: Mona Siddiqui, MD, MPH; Division of General Internal MedicineJohns Hopkins University School of Medicine, Hampton House, Room 626624 North Broadway, Baltimore, MD 21205-1901, USA (e-mail: msiddiq2@jhmi.edu).

\section{REFERENCES}

1. Spicknall KE, Zirwas MJ, English JC 3rd. Clubbing: an update on diagnosis, differential diagnosis, pathophysiology, and clinical relevance. $J$ Am Acad Dermatol. 2005;52(6): 1020-8.

2. Dever LL, Matta JS. Digital clubbing in HIV-infected patients: an observational study. AIDS Patient Care STDS. 2009;23(1):19-22.

3. Moyer VA. Screening for HIV: U.S. preventive services task force recommendation statement. Ann Intern Med. 2013;159:51-60. 